\title{
Experiencias de adolescentes con diabetes mellitus tipo I del estado de Yucatán. Una perspectiva fenomenológica
}

\author{
Experiences of adolescents with type I diabetes mellitus Yucatan state. A \\ phenomenological perspective
}

Argelia Lizandra López Osorio

Elia María Escoffié Aguilar

Manuel Sosa Correa

Universidad Autónoma de Yucatán

\section{Resumen}

La diabetes es una enfermedad que ha aumentado su prevalencia en los últimos años y es considerada uno de los grandes problemas mundiales de salud (Martínez Chamorro, Lastra Martínez, \& Luzuriaga, 2002). Los adolescentes con diabetes resultan ser focos rojos de atención entre la población que padece la enfermedad de diabetes, pues además de trabajar en su adaptación y aceptación del padecimiento, es necesario hacerlo junto con los cambios que presentan debido a su etapa de desarrollo. El presente estudio tiene el objetivo de describir las experiencias de vida de los adolescentes con diabetes mellitus tipo I de una asociación civil del estado de Yucatán; desde sus propias narraciones e historias y desde la voz de actores relacionados. La contextualización se llevó a cabo a través de una fenomenología, con base en técnicas como talleres participativos, entrevistas grupales no estructuradas y entrevistas individuales semiestructuradas. Participaron 16 jóvenes con diabetes mellitus tipo I, de los cuales nueve son hombres y siete son mujeres, cuyas edades se encuentran entre los 13 y 17 años; seis padres de familia, dos hombres y cuatro mujeres. La muestra la conforma personal de la Asociación civil y personal de salud que funciona como consultor externo. Fue posible observar que los diferentes actores relatan aspectos similares de índole psicológica. Sin embargo, destacan los discursos de los adolescentes, los cuales reflejan la saturación del poder: el poder médico que les dice cómo regularse, de los padres que insisten en un fuerte cuidado y de una sociedad que los señala y rechaza por su condición de vida.

Palabras clave: diabetes tipo I, adolescentes, experiencias, fenomenología, asociación civil.

Nota del autor

Argelia Lizandra López Osorio, Facultad de Psicología, Universidad Autónoma de Yucatán (UADY); Elia María Escoffié Aguilar, Facultad de Psicología, UADY; Manuel Sosa Correa, Facultad de Psicología, UADY.

La correspondencia en relación con este artículo debe dirigirse a Argelia Lizandra López Osorio, Facultad de Psicología, UADY, carretera Mérida-Tizimin, Km 1, Cholul. Mérida, Yucatán, México.

Dirección electrónica: argelia.lopez.osorio@gmail.com 


\begin{abstract}
Diabetes is a disease that has increased in prevalence in recent years and is considered one of the major global health problems (Martinez Chamorro, Luzuriaga Lastra Martinez, \&Thomas, 2002). Adolescents with diabetes appear to be red flags among people with diabetes, sinace, s well as working their adaptation and acceptance of the disease, it is necessary to do it with the changes that occur due to their stage of development. This study aims to describe the life experiences of adolescents with diabetes mellitus type I of a civil association of Yucatan state; from their own narratives and stories and from the voice of related actors. Contextualization was carried out through a phenomenology, using techniques participatory workshops, group interviews unstructured and semi-structured individual interviews. They involved 16 young people with type I diabetes mellitus, of which 9 are men and 7 are women, whose ages are between 13 and 17; 6 parents, 2 men and 4 women; civil staff and staff Health Association works as an external consultant. It was observed that the different actors relate similar aspects of a psychological nature. However, they highlight the speeches of adolescents, which reflect the saturation power, medical power that tells them how regulated, parents who insist on a strong care and a society that states and rejects their living conditions.
\end{abstract}

Keywords: diabetes type I, teenagers, experiences, phenomenology, civil association.

Las enfermedades crónicas son enfermedades de larga duración $\mathrm{y}$, por lo general, de progresión lenta. En 2008, 36 millones de personas murieron de una enfermedad crónica (Organización Mundial de la Salud, 2015). En los últimos años, la diabetes ha aumentado su prevalencia, al respecto, Martínez-Chamorro, Lastra-Martínez y Luzuriaga Tomas (2002), la consideran uno de los grandes problemas mundiales de salud y una enfermedad social, no sólo por su elevada frecuencia, sino también por su gran coste económico. Asimismo, nuestro país presenta un alto índice de personas con diabetes; $73 \%$ de las muertes en México se debe a padecimientos no transmisibles, como la diabetes, los problemas cardio-vasculares y el cáncer (Secretaría de Salud, 2007). El estado de Yucatán, a su vez tiene grandes retos en materia de enfermedades crónicas, especialmente relacionados con la diabetes, según lo señala su Plan Estatal de Desarrollo 2012-2018 (Gobierno del Estado de Yucatán, 2013), pues el número de personas que la padece aumenta cada año. La diabetes mellitus tipo I (insulino dependiente) es uno de los desórdenes endocrinos crónicos más comunes en la niñez y la adolescencia (Salvador-Ortiz, 2004); de acuerdo con la Federación Internacional de Diabetes (FID, 2013), el número de niños y adolescentes que presenta diabetes mellitus tipo I (DM1) aumenta cada vez más rápido.

Por otro lado, las personas cargan con adscripciones o etiquetas impuestas en lo social o autoimpuestas para categorizarse o incluirse en relación a los demás. Estas categorizaciones a veces dependen de su sexo, edad, color de piel, 
origenétnico, orientación sexual, profesión, clase social, nivel de estudios, estado civil, religión, etc. Así sucede en la adolescencia, pues como señala Téllez (2013), la identidad adolescente pasa inevitablemente por una influencia cultural. Para Fexia (2011), los adolescentes actuales:

Son seres artificiales, medios robots y medios humanos, escindidos entre la obediencia a los adultos que los han engendrado y la voluntad de emanciparse, adheridos a un modelo de inserción "virtual" en la sociedad, cuyos rasgos característicos son las transiciones discontinuas hacia la edad adulta, la infantilización social, el retraso permanente en el acceso al trabajo y a la residencia, la emergencia de mundos artificiales como las comunidades de internautas y la configuración de redes adolescentes a escala planetaria. (p.23).

Como puede observarse, resulta muy importante trabajar con diabetes mellitus tipo I y, en especial, con los adolescentes que la presentan, quienes además de asumir el reto de adaptarse a los desafíos socio-culturales impuestos para su edad, deben asumir su autocontrol (realización de autoanálisis y toma de decisiones), prestar atención constante a la dieta, ingesta de alcohol, actividades físicas, etc., es decir, el adolescente ha de hacer frente a una serie de aspectos que le hace diferente de sus compañeros (Martínez-Chamorro et al., 2002).

Los adolescentes con diabetes mellitus se encuentran en una posición de subordinación ante los actores relacionados con su cuidado: médicos, enfermeros, nutriólogos y demás personal de salud, al igual que de padres de familia y otros familiares. La insistencia $y$ presión respecto a su autocontrol y adherencia al tratamiento, en ocasiones, resulta ser la plática de todos los días, dejando de lado lo que los jóvenes son, piensan y sienten. El objetivo de este estudio es describir las experiencias de vida de los adolescentes con diabetes mellitus tipo I de una asociación civil del estado de Yucatán, desde sus propias narraciones e historias y desde la voz de actores relacionados, tales como sus cuidadores, personal de la asociación y personal de salud. Al respecto, Payne (2002) menciona que ante problemas graves o potencialmente extremos la idea de escuchar puede parecer trivial, pero las conversaciones crean realidades nuevas. La vida de cada uno de nosotros es una historia construida, donde el actor principal del relato es la persona que nos está relatando los acontecimientos (Zlachevsky, 2003). Los puentes de significado que se crean hacen que se produzcan resultados curativos (Freeman, Epston, \& Lobovits, 2010).

La intención de la presente investigación de corte cualitativo, es conocer dichas experiencias bajo el enfoque de los sujetos participantes, los principales actores del relato, no solamente bajo aspectos patológicos y discursos de poder, a los cuales los adolescentes con diabetes están tan acostumbrados. La investigación cualitativa posibilita que el investigador capte la reacción de los individuos en su propia realidad. Se 
trabaja con el universo de significados, valores y actitudes de los individuos, de modo que el investigador conocer la dinámica y la situación desde el punto de vista de quienes la vivencian (Ramírez, Ángelo, \& González, 2011).

Específicamente se ha observado que al buscar la conciencia y los significados a través de la investigación fenomenológica, las necesidades y potencialidades de los individuos se dan a conocer mejor, y se abren vías importantes para la realización de cambios en los procesos de cuidados de los pacientes, en este caso adolescentes con diabetes mellitus tipo I (Pavan, Barbosa, \& Fernandes, 2011). Al recabar la vivencia de éstos, es posible al personal de salud, brindar una atención más adecuada, sobre todo, humana, fundamentada en la importancia de las personas, quienes narrarán sus experiencias. Esto permite investigar tanto los comportamientos individuales como de grupos sociales que viven una situación típica; como diría Schütz (citado en Dreher, 2012), el mundo de la vida cotidiana, el cual define de la siguiente manera:

Que es desde el principio un mundo social y cultural dentro del cual el sujeto se relaciona, de múltiples formas de interacción con los semejantes que se conocen en diversos grados de intimidad y anonimato. Este trabajo permite investigar y conocer el mundo de la vida cotidiana de los adolescentes con diabetes mellitus tipo I y actores relacionados con estos. (p77).
Con lo anterior, en el contexto de salud, al tratar de comprender el significado de la experiencia vivida por seres humanos, emergen contribuciones valiosas para las múltiples dimensiones involucradas en el cuidado de la vida humana, hasta ahora inexploradas (Pavan et al., 2011).

\section{Método}

La contextualización se llevó a cabo a través de una fenomenología, ya que este método se consideró más adecuado, pues permite tener un acercamiento ala población conlaque se trabajará de manera poco invasiva. La fenomenología quiere entender los fenómenos sociales desde la propia perspectiva del actor; la realidad que importa es la que las personas perciben como importante (Galeano, 2004). Su propósito es estudiar el pensamiento y las acciones humanas, describir la esencia de la experiencia vivida. Busca la conceptualización del mundo de la vida, de los hechos y situaciones que enfrenta el hombre en su vida cotidiana (Rodríguez, 2014). La fenomenología pretende la comprensión en un nivel personal de los motivos y creencias que están detrás de la gente (Galeano, 2004).

Con esta metodología, se pretendió conocer la experiencia de vida de los adolescentes con diabetes mellitus tipo I, sin que éstos se sintieran cuestionados o estudiados. Se buscó que este acercamiento fuera lo más natural posible, pues al estar expuestos a diferentes profesionales de 
la salud, chequeos y hospitales, los adolescentes se sienten saturados y cansados.

\section{Participantes}

Se realizó una contextualización con diversos actores directamente relacionados con una asociación civil encargada del trabajo de niños $\mathrm{y}$ adolescentes con diabetes mellitus tipo I del estado de Yucatán. En dicha contextualización participaron 16 jóvenes, de los cuales ocho son hombres y ocho son mujeres, cuyas edades se encuentran entre los 13 y 17 años. Estos jóvenes son usuarios regulares de los servicios que brinda dicha asociación; la mayoría radica en la ciudad de Mérida, aunque cinco de ellos viven en comunidades del interior del estado o comisarías de Mérida. En general, el nivel socioeconómico del grupo es de medio a medio bajo.

De igual forma, participaron en esta aproximación seis padres de familia de los jóvenes antes mencionados, tres son hombres y tres mujeres; la coordinadora general de la asociación, quien es nutrióloga de profesión y lleva cinco años al frente de la misma; la pasante de nutrición en turno, encargada del trabajo de educación en diabetes con los jóvenes; una nutrióloga; y una psicóloga. Estas últimas, aunque no pertenecen a la asociación civil, funcionan como consultoras externas de ella $\mathrm{y}$ han atendido en varias ocasiones a jóvenes referidos por la asociación.

\section{Instrumentos}

Las técnicas que se utilizaron durante el diagnóstico fueron la entrevista grupal no estructurada, entrevistas individuales semiestructuradas y taller participativo.

La entrevista grupal no estructurada se llevó a cabo con los padres de familia. Esta técnica se eligió debido a que da gran libertad a los entrevistados para poder hablar sin límites acerca de un tema determinado. Ander-Egg (2003) menciona que esta entrevista se trata, en general, de un listado de preguntas abiertas que son respondidas dentro de una conversación, teniendo como característica principalla ausencia de una estandarización formal. También refiere que la persona interrogada responde de forma exhaustiva, con sus propios términos y dentro de su cuadro de referencia, a la cuestión general que le ha sido formulada. Con dicha técnica, los padres de familia tuvieron oportunidad de hablar de sus experiencias, de contar y narrar sus historias sin sentirse atados en sus relatos y con la libertad de expandirse hasta el punto que consideraran necesario.

Las entrevistas individuales semiestructuradas se aplicaron a los profesionales de la salud, tanto de la asociación como a los consultores externos. Se eligió este tipo de entrevista por ser, también, de carácter flexible. AnderEgg (2003) refiere que lo fundamental de esta técnica es el guion de temas y objetivos que se consideran relevantes para la investigación, lo cual otorga un amplio margen de libertad al en- 
trevistado.

El tipo de conversación producida en la entrevista brinda información que puede ser comprendida en el contexto de un relato, el cual tiene que ser reconstruido una y otra vez en el marco de las condiciones de enunciación y en relación con comentarios pasados y futuros; el texto producido mediante la situación de entrevista representa el universo social de referencia de la persona entrevistada y permite captar mediante sucesivas lecturas y relecturas los distintos elementos que componen su mundo significativo, la visión de sí mismo, la emergencia de discursos que relacionan al entrevistado con sus grupos de referencia, entre otros aspectos (Vargas, 2012). Las narrativas presentes en la entrevista representan descripciones de la organización de las actividades de las personas, y en ese carácter descriptivo radica la potencialidad de captar fuentes de significado (Merlisnky, 2006).

Finalmente, se realizó un taller participativo con los adolescentes, el cual constó de dos sesiones de dos horas cada una. La International HIV/AIDS Alliance (2002) consideró adecuado el uso de metodologías participativas en situaciones donde un grupo de personas debe trabajar en conjunto para resolver un problema común, ya que se requieren las experiencias y conocimientos, de una serie de personas. Con estas técnicas se facilita compartir experiencias y conocimientos, así como se demuestra que la contribución de cada persona es importante.

Durante el taller se realizaron actividades de juego que ayudaron a los chicos a sentirse más en confianza y disminuir el estrés durante el trabajo en grupo. Asimismo, las técnicas de juego permitieron a los participantes contar sus historias de una forma menos amenazante y más extensa. Al respecto, Schaefer (2012) menciona que la persona es el autor de la historia, sólo hay que proporcionarle el espacio de reescribirla, en ese sentido, el taller participativo fue un buen espacio para comenzar con la narración de sus propias historias.

\section{Procedimiento}

La entrevista grupal no estructurada se aplicó a los padres de familia de los adolescentes con diabetes mellitus de la asociación. Se convocó de manera abierta a los padres que quisieran asistir y participar en dicha charla. Acudieron seis personas, tres hombres y tres mujeres, quienes hablaron de manera fluida de sus historias y sus experiencias al enterarse que sus hijos tenían diabetes mellitus tipo I. También platicaron sobre cómo han llevado este proceso y qué observan en sus hijos a raíz del diagnóstico.

Las entrevistasindividualessemiestructuradas fueron respondidos por la coordinadora de la asociación, la pasante de nutrición que trabajaba con los adolescentes, una nutrióloga y una psicóloga de un centro del ayuntamiento de Mérida, quienes funcionan como consultoras externas de la asociación. La duración de cada entrevista fue de 60 a $90 \mathrm{~min}$. Los ejes centrales de la entrevista fueron la experiencia como personal de salud a cargo de la atención de los 
jóvenes, las necesidades que observan en ellos y los factores que, en su experiencia, se relacionan con dichas necesidades.

El taller participativo se realizó en dos sesiones de dos horas. Se efectuaron actividades lúdicas que permitieron la apertura de los jóvenes para narrar sus historias, tales como las máscaras y la botella. En la primera actividad, ellos confeccionaron una máscara propia, agregándole los elementos que desearan para luego presentarse a través de ella frente al resto del grupo y explicar el porqué de los elementos. La segunda actividad sirvió para que los adolescentes hablaran en particular de situaciones relacionadas con la diabetes y cómo ellos viven día a día dicha enfermedad. La dinámica del juego fue la ya conocida: los participantes se sientan en círculo y al centro de ellos se gira una botella, la persona a quien se dirige la parte gruesa de la botella realizará una pregunta, la persona señalada por la parte delgada responderá dicha pregunta. Es importante señalar que después de algunas preguntas el juego pasó a segundo término y la socialización de historias tomó el rol principal. Asimismo, se realizaron registros de temperatura grupal al inicio y al final de cada sesión, y algunas otras dinámicas de juego con el fin de favorecer el ambiente de confianza para la conversación. De igual forma, se llevó a cabo una actividad de análisis y síntesis para especificar y jerarquizar las temáticas que fueron señaladas en sus historias.

\section{Resultados}

Los relatos contados durante la presente fenomenología evidencian las distintas historias que los actores tienen del mismo suceso. Es posible observar los matices, las percepciones y experiencias de cada parte en este mundo de la vida cotidiana.

Llama la atención el primer tema que los adolescentes señalan en sus discursos, y destacan como punto principal para trabajar en un futuro: su integración como grupo, con ello, se muestra la relevancia de pertenecer a un grupo que los cobije y funja como una fuerte red de apoyo para cada uno de ellos, pues al experimentar situaciones similares, las experiencias que se comparten, así como la escucha empática resultan ser bases fundamentales para el camino que recorrerán a lo largo de sus vidas.

Por otro lado, los repetidos, rutinarios $\mathrm{y}$ exigentes cuidados que la diabetes mellitus requiere, ponen en jaque la relación familiar, al crear conflictos entre padres e hijos. Los adolescentes se muestran cansados de la presión $y$ el fuerte cuidado que sus padres depositan sobre ellos. Martínez-Chamorro et al., (2002), mencionan que la aparición de diabetes insulinodependiente en un niño prepúber hace que recaiga en los padres la responsabilidad del tratamiento ambulatorio, lo cual lleva a los padres a aumentar el control sobre el niño, guardándole dentro de una zona de seguridad más o menos estrecha; dichos cuidados continúan hasta la adolescencia, pero éstos ya no son tan bien aceptados por los jóvenes. Al respecto un 
participante comenta: "Es que siempre es, tienes diabetes, tienes diabetes, tienes diabetes, eh, no porque tienes diabetes, no salgas porque tienes diabetes, no corras porque tienes diabetes" (Luis, sesión 2 de taller participativo, 28 de noviembre, 2015).

De igual forma los padres se presentan con preocupaciones frecuentes acerca del tratamiento de sus hijos, ya que a pesar de que ellos "son expertos" en el mismo, manifiestan poca adherencia a éste poniendo en riesgo su salud. Los miedos a que sus hijos tengan complicaciones o mueran son constantes, sobre todo cuando los adolescentes tienen poco tiempo de haber sido diagnosticados o si pasaron por un proceso de internamiento antes de dicho diagnóstico. Estas dificultades familiares comienzan a presentarse de manera cíclica, es decir, los padres presionan porque los hijos no realizan su tratamiento al pie de la letra y los hijos no realizan su tratamiento por la presión de los padres. Los adolescentes se muestran cansados de la situación que viven $\mathrm{y}$, en ocasiones, tiene momentos de escape y rebeldía. A veces dichos momentos se dan cuando se encuentran enojados con sus padres, como una forma de castigo hacia ellos. En este sentido una participante comenta:

El viernes fui a un cumpleaños y había una mesa de dulces y como era dama nos dieron un taloncito y comí de todos los dulces que habían en la mesa ... fue en ese momento en el que estaba yo molesta con mi mamá y me regañó... Odio que me peinen y fue así como que: ¿no vas a ir a que te peinen? y llegué a la fiesta y comí un dulce de cada uno... fue terrible... y en la noche que me checaron tenía 500 y amanecí con 500 y seguí con $500 \ldots$ y luego me tuve que inyectar sin que lo vea mi mamá y luego fue que lo bajé a 50 y ya... luego te sientes mal porque recuerdas que al final la única que se hace daño eres tú y ahí es cuando lloro y más como. (Aurora, sesión 2 de taller participativo, 28 de noviembre, 2015).

Por otro lado, lo atareado de la vida actual, la situación económica y el elevado costo del tratamiento para la diabetes, lleva a ambos padres a trabajar, por lo cual, muchas veces, los hijos se quedan al cuidado de la familia extensa, situación que ocasiona conflictos entre los padres de familia y los demás cuidadores, debido a los acuerdos o la carencia d éstos respecto al seguimiento del tratamiento del adolescente. Al respecto la psicóloga entrevistada comenta:

Se crean problemas hasta en cuestión macro, porque se vuelve una razón más para que se creen problemas con la familia. La salud física depende del cuidado que se tenga con él ¿Checaste que se pinche, cuánto se sacó? Que si le corresponde al abuelito a la abuelita porque son los que están al cuidado... y la salud depende de esto... una excusa para que se involucren más. (Entrevista realizada a Regina, 3 de diciembre, 2015).

Asimismo, los desacuerdos y la fuerte responsabilidad que conlleva estar a cargo de 
un hijo con diabetes, mellitus tipo I, genera dificultades en las parejas al grado de llevarlos en ocasiones al divorcio. Las madres refieren menos compromiso de parte de sus esposos, por lo que la mayor parte de la responsabilidad recae sobre la figura materna. Ellas comentan que en muchas ocasiones a sus parejas les es complicado aceptar la situación de vida de sus hijos. En este sentido Carmen menciona:

Yo tengo mucho problema con mi niña porque mi esposo la consiente... mi esposo ya le dijeron que esta propenso a una embolia, un derrame... Le vale... Mi esposo cree que es chiste... él cuando puede llega con un pan y ni modo que le diga que no lo coma si lo está viendo...me siento sola... mi esposo no me apoya... cómo le digo que no lo haga si está viendo que su papá lo hace... Entonces dirá que mi mamá es la mala... (Carmen, madre de Aurora, en entrevista grupal, 5 de diciembre, 2015).

Por otro lado, las emociones de los adolescentes y la influencia de éstas en sus niveles de glucosa parece ser un punto central que pasa desapercibido en la persecución de la adherencia al tratamiento biomédico. Algunas investigaciones han determinado que existe una relación entre eventos estresantes de la vida y el control de la glucemia en diabéticos (Pineda et al., 2004). Los cambios continuos del estado de humor y emociones de los adolescentes, traen consigo variaciones repentinas en sus niveles de glucosa. Situaciones comunes que se presentan en su vida diaria les ocasionan hipoglucemias o hiperglucemias; no existe necesariamente un patrón común para la emoción y el resultado que presentan. En este sentido los participantes refieren:

J- ¿Cuándo estás con la persona que te gusta qué te pasa? ¿Se te sube o se te baja tu glucosa? ¿Te pasa algo... se te sube?

L- No. Cuando me molesto... son las hormonas... se me baja... a veces ni me puedes ver porque te mando muy lejos... se me baja.

$\mathrm{K}$ - A mí se me baja.

P- Me checo y estoy como a 120, mi azúcar se vuelve loca. Se sube, se baja. A mí solamente cuando me someten a mucha adrenalina... me empiezo a sentir mal, pero no sé si estoy alta o baja.

A- Es como ansiedad... yo tenía proyectos en la escuela y llegaba a mi casa almorzaba, me metía a bañar y ya tengo hambre, pero como estoy estresada... ni siquiera te tiene que gustar...

O- Cuando tengo ansiedad me checo y tengo 140 cambio el resultado... sólo cuando tengo ansiedad... así mucha... y después me inyecto. (Juan, Luis, Kevin, Patty, Aurora y Orlando, sesión 2 de taller participativo, 28 de noviembre, 2015).

Los adolescentes con diabetes mellitus tipo I, tienen que monitorearse constantemente e inyectarse de dos a tres veces por día. En ocasiones, estos procesos son llevados a cabo delante de sus compañeros de escuela, maestros, 
amigos, familiares, etc. El desconocimiento de este proceso por parte de los observadores puede ocasionar discriminación de los adolescentes con diabetes, quienes muchas veces, por temor, prefieren mantener oculto su padecimiento. Murray (2009, en Ledón-Llanes, 2012) afirma que muchas comunidades tienen actitudes, creencias y políticas desfavorables hacia las personas con diabetes. Asimismo, menciona que la ignorancia y el temor son las principales causas de discriminación, aunque las creencias culturales también juegan un rol importante. Los adolescentes refieren ocultar su condición, la mayoría de las veces al entablar nuevas relaciones interpersonales, y con el paso del tiempo, cuando la relación es más estable, revelarla. Acerca de esto, mencionan:

L- ¿Cuándo supiste que tenías diabetes y llegaste a la escuela no te daba miedo que las personas lo supieran?

K- No. La verdad sí. La neta sí, pero ya lo dijo la maestra.

P- En mi escuela lo vocearon... porque falté, no fui una semana y en el homenaje dijeron, bueno pues vamos a rezar por "C" porque tiene diabetes. (Luis, Kevin y Patty, sesión 2 de taller participativo, 28 de noviembre, 2015).

Los participantes afirman que las discriminaciones son constantes y los desalientan, pues se han dado en varios ámbitos, como en deportes, escuela e incluso en relaciones de noviazgo. A lo anterior se relaciona con complicaciones con la autoestima de los adolescentes con diabetes mellitus, quienes prefieren ocultar su condición de salud para no ser señalados, lo cual trae consigo riesgos importantes, pues los jóvenes en ocasiones deciden salir sin sus glucómetros o prefieren no avisar cuando se sienten mal y están fuera de casa. Asimismo, los adolescentes tienden a no seguir las indicaciones en cuanto a consumo de alimentos cuando se encuentran entre sus pares. Esto se puede observar claramente en un relato realizado por una madre participante; el enamorado de su hija le comentó que tenía prohibido salir con ésta, debido a la diabetes mellitus: "Sabe por qué no quiere mi mamá que yo ande con Aurora, porque es diabética, dice mi mamá que por qué ando con una enferma..." (Carmen, madre de Aurora, en entrevista grupal, 5 de diciembre, 2015).

Por otro lado, el servicio médico y la atención de especialistas es un punto importante en esta investigación. Gran parte del tiempo de los adolescentes y sus familias lo pasan con personal de salud: endocrinólogos, nutriólogos, químicos, enfermeros, son personajes cotidianos en el devenir de los participantes, y que sin duda alguna marcan gran parte de sus experiencias y discursos. Como se mencionó con anterioridad, el coste del tratamiento de la diabetes mellitus es elevado, por lo cual la mayoría de los adolescentes acude a un servicio médico público. Sin embargo, tanto las personas que asisten a un servicio público como a uno privado refieren que la atención del personal de salud es fría y distante. 
Los médicos y las enfermeras son vistos como duros y poco comprensivos con la situación que viven los adolescentes con diabetes mellitus y sus familias; son percibidos como personas renuentes a brindarles información completa de la enfermedad, tratamientos o cuidados, por consecuencia, se dificulta la adherencia al tratamiento de los adolescentes. El trato recibido por parte de su endocrinólogo de cabecera resulta ser decisivo en el seguimiento del tratamiento. Los padres de familia y los mismos adolescentes se refieren con cariño o rechazo a diferentes médicos de la comunidad yucateca, que los han apoyado y guiado en este proceso o que, por el contrario, los han dejado solos en este barco, como ellos mencionan. En este sentido Soria, Vega, Nava y Saavedra (2011) resaltan la importancia de la relación entre el médico y el paciente, como factor que influye en el control de las enfermedades crónicas, en tanto, la falta de compromiso del médico hacia sus pacientes como un factor que merma la adherencia terapéutica, provoca la prolongación del tratamiento. Al respecto, Adriana relata:

Es muy difícil todo esto al principio, te cae como bomba... comienzos a buscar culpables... me dijeron que era el estrés de los exámenes... después de un mes se presentó todo un caos, llegó como a 560' pero todo descompensado... No funcionaba lo que le hacían en el seguro... y lo tuvieron que internar, él lo tomó bien... nosotros sólo llorar y llorar... una enfermera salió y me dijo 'ay, señora por favor deje de llorar, usted lo que tiene que hacer es buscar información y levantar a su hijo'... ¿Y dónde? 'Pues no sé... usted tiene que buscarla'. (Adriana, madre de Joaquín, en entrevista grupal, 5 de diciembre, 2015).

Finalmente, los adolescentes remarcan en sus historias la necesidad de una vida sin diabetes y no en el sentido de desaparecer esta condición de su vida, sino más bien, que las demás personas, familiares, amigos, personal de salud, cuidadores, etc., puedan ver todo lo que ellos son $\mathrm{y}$ pueden hacer, sin necesariamente relacionarlo con la diabetes. Patty menciona al respecto: "No sólo soy diabetes, yo dibujé cosas positivas, lo que me gusta hacer y lo que soy, no hablé de la diabetes (en su dibujo) porque no sólo soy eso" (Patty, sesión 1 de taller participativo, 7 de noviembre, 2015).

\section{Discusión}

La fenomenología permitió, en esta ocasión, conocer las experiencias de los adolescentes con diabetes mellitus tipo I, de una forma profunda, con énfasis en la importancia de las narraciones y de los relatos de las personas desde su propia vivencia, lo cual enriquece sobre manera el trabajo realizado en salud. Seguramente, la presente investigación no encajará en otros tipos de estudios utilizados en el área de salud y desde el modelo biomédico, en el cual se busca conocer una realidad objetiva y generalizable. Sin embargo, permitió conocer un poco más las 
diferentes historias de un grupo de personas, cuyas realidades y discursos resultan ser tan válidos y aplicables para ellas en este momento de vida, como lo sería la realidad objetiva en estudios positivistas.

El modelo biomédico, sin duda, presenta discursos saturados de poder, que miran a las personas con diabetes mellitus como enfermos, los cuales deben ser guiados en su proceso de enfermedad para la constante búsqueda de salud o, en el caso de las personas con enfermedades crónico-degenerativas, para su control o regulación. En este contexto, la cultura biomédica expresada a través del sistema médico occidental, se ha establecido mundialmente como el modelo capaz de resolver, si no todos, la mayoría de los problemas de salud de la población con independencia de los contextos sociales y culturales en que se desarrolla la enfermedad (Alarcón, Vidal, \& Neira, 2003).

Estos discursos se observan claramente en las narraciones de los adolescentes, al igual que en sus reacciones de hastío hacia los mismos. Los adolescentes con diabetes mellitus quieren ser percibidos desde otra perspectiva, con anteojos diferentes; exigen ser vistos con la diabetes a su lado, como una compañera y no unida a ellos mismos. Por ello, esta exploración fenomenológica sienta bases para futuras investigaciones en materia de salud, que generen intervenciones más equitativas y planteadas en igualdad de circunstancias, que analicen y tomen en cuenta estas vivencias recabadas y que busquen eliminar las relaciones de poder impuestas en nuestra sociedad, nuestras familias y los sistemas de salud. Especialmente en estos últimos, pues en ellos se veneran ciertas profesiones y se jerarquizan las relaciones establecidas entre pacientes y personal de salud, al tiempo que hacen a un lado las vivencias, experiencias y necesidades expresadas por las personas con diabetes mellitus.

En sí, los estudios fenomenológicos nos permiten mirar la realidad construida desde otra perspectiva y observar las concepciones que pequeños grupos realizan de su propia realidad, la cual no concuerda necesariamente con la dominante, como en este caso. Lo anterior se evidencia cuando los adolescentes señalaron como tema principal a abordar en intervenciones futuras: la integración como grupo, lo cual de seguro es contrario a lo que los demás actores perciben como principal temática a abordar en el trabajo con adolescentes con diabetes mellitus.

Lo anterior deja ver la importancia de realizar intervenciones, desde la comprensión y apertura, y no desde un punto de expertez y jerarquía, que generen relaciones horizontales basadas en diálogos abiertos, de aceptación y de respeto. Lo dicho con anterioridad acontece en el ámbito de una relación completamente simétrica entre el sujeto, su familia y el equipo de salud (Builes \& Bedoya, 2006). Sin duda, es un camino largo por recorrer, pero se considera necesario comenzar a picar piedra en modelos de intervención en materia de salud, pues estas intervenciones co-construirán nuevas historias y nuevas alternativas junto con las personas con 
una enfermedad crónico-degenerativa como lo es la diabetes.

De igual forma, se considera fundamental el trabajo psicológico en esta área. Martín, Querol, Larsson, y Renovell (2007) señalan que la mejora de la calidad de vida de los pacientes con diabetes a través del procedimiento de intervención psicológica, ha demostrado su utilidad y eficacia en los trastornos relacionados con la medicina del comportamiento. Sin embargo, se considera que dichas intervenciones psicológicas se deben realizar desde enfoques con miradas abiertas, con prácticas distintas, que exploren en conjunto las historias de las personas y tiendan puentes, sobre todo, que no pierdan de vista que, al final, el experto en su vida siempre será el cliente. Donde se haga hincapié en el respeto por la forma de entender del sistema consultante, considerando todo el tiempo que es el paciente quien sabe de sí mismo (Zlachevsky, 2003).

\section{Referencias}

Alarcón, M., Vidal, H., \& Neira, J. (2003). Salud intercultural: elementos para la construcción de sus bases conceptuales. Revista Médica de Chile, 131(9), 1061-1065. Recuperado de https://dx.doi.org/10.4067/S0034-98872 003000900014

Ander-Egg, E. (2003). Métodos y técnicas de investigación social IV. Técnicas para la recogida de datos e información. Buenos Aires: Lumen.
Builes, M., \& Bedoya, M. (2006). La psicoeducación como experiencia narrativa: comprensiones posmodernas en el abordaje de la enfermedad mental. Revista Colombiana de Psiquiatría, 35(4), 463-475.

Dreher, J. (2012). Fenomenología: Alfred Schütz y Thomas Luckman. En E. De la Garza y G. Leyva (2012): Tratado de las ciencias sociales: perspectivas actuales. México: Universidad Autónoma Metropolitana y Fondo de Cultura Económica. Recuperado de http://www. socialsciencesmeditationnews. org/ weblog/wp-content/uploads/2013/08/ Fenomenologia-y-Sociologia-Drecher. pdf

Federación Internacional de Diabetes. (2013). ATLAS de la Diabetes de la FID $6^{a}$ edición. Recuperado de www.idf.org/diabetesatlas

Fexia,C.(2011).Unidosporelflog: ¿Ciberculturas juveniles? Revista Nuevas Tendencias en Antropología, (2), 16-36. Recuperado de http://www.revistadeantropologia.es/Textos/ N2/Unidos\%20por\%20el\%20Flog.pdf

Freeman, J., Epston, D., \& Lobovits, D. (2010).

Terapia narrativa para niños. Aproximación a los conflictos familiares a través del juego. Madrid: Paidós.

Galeano, M. (2004). Diseño de proyectos en la investigación cualitativa. Medellín: Universidad EAFIT.

Gobierno del Estado de Yucatán. (2013). Plan Estatal de Desarrollo 2012-2018. Mérida, México: Impresión de autor.

International HIV/AIDS Alliance. (2002). Una Guía para Facilitadores de Talleres 
Participativos con ONGs/OBCs que Trabajan en VIH/SIDA. Recuperado de http:// www.aidsalliance.org/assets/000/001/048/ fgs0302_Facilitators_guide_sp_original. pdf? 1413459083

Ledón-Llanes, L. (2012). Psychosocial impact of diabetes mellitus, experiences, meanings, and responses to disease. Revista Cubana de Endocrinología, 23(1), 7697. Recuperado de http://scielo.sld.cu/ scielo.php?script $=$ sci_arttext\&pid $=\mathrm{S} 1561$ 29532012000100007\&lng= es\&tlng=en

Martín, E., Querol, M., Larsson, C., \& Renovell, C. (2007). Evaluación psicológica de pacientes con diabetes mellitus. Diabetología, 23(2), 88-93. Recuperado de http://www.sediabetes.org/gestor/upload/ revistaAvances/23-2.pdf\#page $=18$

Martínez-Chamorro, M., Lastra-Martínez, I., \& Luzuriaga, C. (2002). Perfil psicosocial de niños y adolescentes con diabetes mellitus. Boletín de la Sociedad de Pediatría de Asturias, Cantabria, Castilla y León, 42(180), 114-119.

Merlinsky, G. (2006, diciembre). La entrevista como forma de conocimiento y como texto negociado: notas para una pedagogía de la investigación. Revista Cinta de Moebio: Revista Electrónica de Epistemología de Ciencias Sociales, 27, 27-33. Recuperado de http://dialnet. unirioja.es/servlet/listaarticulos?tipo_ busqueda $=$ EJEMPLAR\&revista_busqu eda $=2197 \&$ clave_busqueda $=157274$
Organización Mundial de la Salud. (2015). Temas de salud. Enfermedades crónicas. Recuperado de http://www.who.int/topics/ chronic_diseases/es/

Pavan, P., Barbosa, M., \& Fernandes, G. (2011). Fenomenología. El estudio de la fenomenología como una vía de acceso a la mejora de los cuidados de enfermería. Cultura de los cuidados, 15(29), 9-15. Recuperado de http://www.index-f.com/ cultura/29pdf/29-009.pdf

Payne, M. (2002). Terapia narrativa. Una introducción para profesionales. Madrid: Paidós.

Pineda, N., Bermúdez, V., Cano, C., Ambard, M., Mengual, E., Medina, M., Leal, E., Martínez, Y., \& Cano, R. (2004). Aspectos psicológicos y personales en el manejo de la diabetes mellitus. Archivos Venezolanos de Farmacología y Terapéutica, 23(1), 13-17. Recuperado de http://www. scielo.org.ve/scielo.php?script $=$ sci_ar ttext\&pid=S0798-0264200400010000 $3 \& \operatorname{lng}=\mathrm{es} \& \mathrm{t} \operatorname{lng}=\mathrm{es}$

Ramírez, A., Angelo, M., \& González, L. (2011). Vivencia de estudiantes de enfermería de la transición a la práctica profesional: un enfoque fenomenológico social. Texto $y$ Contexto-Enfermagem, 20, 66-73. Recuperado de https://dx.doi.org/10.1590/ S0104-07072011000500008

Rodríguez, M. (2014). Investigación cualitativa. El método de la doble pregunta y las siete llaves del conocimiento cualitativo. 
Recuperado de https://books.google.com. $\mathrm{mx} /$ books? id=RBIFCQAAQBAJ\&printsec $=$ frontcover $\& \mathrm{hl}=\mathrm{es} \# \mathrm{v}=$ onepage $\& \mathrm{q} \& \mathrm{f}=$ false

Salvador-Ortiz, M. (2004). Factores Psicológicos y Sociales Asociados a la Adherencia al Tratamiento en Adolescentes Diabéticos Tipo 1. Psykhe (Santiago), 13(1), 21-31. Recuperado de http://www.scielo.cl/ scielo.php?script $=$ sci_arttext\&pid $=$ S0718$22282004000100002 \& \operatorname{lng}=$ es \& $\operatorname{tln} \mathrm{g}=\mathrm{es}$. 10.4067/S0718-22282004000100002

Schaefer, C. (2012). Fundamentos de terapia de juego (2da ed.). México: Manual Moderno.

Secretaría de Salud de México. (2007). Programa Nacional de Salud 2007-2012. Por un México sano: construyendo alianzas para una mejor salud. México: Impresión de autor.

Soria, R., Vega, Z., Nava, C., \& Saavedra, K. (2011). Interacción médico-paciente y su relación con el control del padecimiento en enfermos crónicos. Liberabit, 17(2), 223230. Recuperado de http://www.scielo.org. pe/pdf/liber/v17n2/a11v17n2

Téllez, A. (2013). El análisis de la adolescencia desde a antropología y la perspectiva de género. Interacciones, 25, 52-73. Recuperado de http://revistas.rcaap.pt/interaccoes/article/ viewFile/2851/23 56

Vargas, I. (2012). La entrevista en la investigación cualitativa: Nuevas tendencias y retos. Calidad en la educación superior, 3(1), 119-139.
Zlachevsky, A. (2003). Psicoterapia sistémica centrada en narrativas: una aproximación. Revista Limite 10. Recuperado de http:// redalyc.uaemex.mx/src/inicio/ArtPdfRed. jsp iCve $=83601003$

Recibido el 11 de noviembre de 2015

Revisado el 13 de noviembre de 2015

Aceptado el 24 de mayo de 2016 